\title{
Oral insulin for diabetes prevention in NOD mice: potentiation by enhancing Th2 cytokine expression in the gut through bacterial adjuvant
}

\author{
B. H artmann, K. B ellmann, I. G hiea, R . K leemann, H . Kolb \\ Clinical Department, Diabetes Research Institute at the Heinrich-Heine-University of Düsseldorf, Düsseldorf, Germany
}

Summary Oral administration of insulin suppresses the development of diabetes in nonobese diabetic (NOD) mice and deviates the cytokine balance in the islets of Langerhans from a Th1 to a Th2 type cytokine pattern. However, the effect of oral insulin is limited and disease suppression is limited to a narrow dose range. Therefore we tried to improve the outcome of suboptimal insulin dosing by bacterial adjuvant. Mice treated with a suboptimal dose of oral insulin showed no change in diabetes incidence although a shift from Th1 towards Th2 cytokine expression occurred in inflamed islets. Significant suppression of diabetes development was only seen in NOD mice receiving both, insulin and the bacterial preparation OM-89 as adjuvant. OM-89 is a protein extract of E scherichia coli, with nonspecific immunostimulatory properties. Potentiation of the effect of oral insulin by the adjuvant was associated with upregulation of interleukin (IL)-4 Th2 cells in infiltrated islets and sustained local IL-2 gene expression. RT PCR analyses of cytokine expression in the gut showed a clear deviation to Th2 type reactivity and downregulation of inducible nitric oxide (NO) synthase (iNOS) expression by the bacterial adjuvant but not by oral insulin alone. Since macrophages are the primary target cells of adjuvant action we tested its effect on mouse macrophages in vitro. Treatment with OM-89 induced transient release of tumour necrosis factor alpha and nitrite but rendered macrophages refractory to restimulation by the potent macrophage activator lipopolysaccharide. In conclusion, the protective effect of oral insulin can be potentiated by pretreatment with the bacterial adjuvant OM-89. This effect correlates with enhanced Th2 cytokine and decreased iNOS gene expression in the gut, probably due to the downregulation of proinflammatory mediators by exposure to the adjuvant. [Diabetologia (1997) 40: 902909]

Keywords Diabetes mellitus, oral tolerance, mucosal immunology, antigen presenting cells, Th2 cytokines.
Insulin-dependent diabetes mellitus (IDDM) or type 1 diabetes is an immune mediated disease with substantial autoimmune phenomena $[1,2]$. A

Received: 28 January 1997 and in revised form 16 April 1997

Corresponding author: Dr. B.Hartmann, Diabetes Research Institute, Auf'm Hennekamp 65, D-40225 Düsseldorf, Germany

A bbreviations: IDDM, Insulin-dependent diabetes mellitus; PSL, ${ }^{52} \mathrm{P}$ stimulated luminescence; NOD, non obese diabetic; IL, interleukin; iNOS, inducible nitric oxide synthase; TGF $\beta$; transforming growth factor beta; LPS, lipopolysaccharide; IFN $\gamma$, interferon gamma.

The first two authors contributed equally to this work corresponding animal model, the nonobese diabetic (NOD) mouse, spontaneously develops a disease with many immunological and pathological similarities to IDDM. In the mouse model the prediabetic phase of the disease is characterized by infiltration of the islets of Langerhans by immune cells like macrophages, T and B lymphocytes. Analysis of infiltrates shows an initial, non-destructive periinsular infiltrate which is characterized by a T-helper type 2 (Th2) associated cytokine pattern [3]. At a later stage, progression towards intrainsular infiltration is seen, during which beta-cell destruction is observed. The destructive insulitis stage is associated with Th1 type immune reactions $[3,4]$. 
Attempts to intervene in the disease process therefore have focussed on supporting "protective" Th2 over "diabetogenic" Th1 reactivities by antigen specific or non-specific treatment modalities [5-8]. Because of an apparently low risk of adverse effects, a promising approach of Th1/Th2 immune deviation is the mucosal administration of antigen. Peroral administration of target autoantigens has been shown to mitigate or suppress Th1 dependent autoimmune diseases such as experimental autoimmune encephalomyelitis or collagen induced rheumatoid arthritis in rodents [9-11]. Dependent on the antigen dose administered there is a deletion or anergy of specific T cells, or an induction of Th2-type antigen reactive $\mathrm{T}$ cells. The latter cells are thought to migrate to the respective target organ, become activated upon re-encounter of antigen and exert bystander suppression of any local Th1 dependent inflammation through their release of interleukin (IL)-4, IL-10, transforming growth factor beta (TGF $\beta$ ) or other Th2 type mediators [12,13]. Deviation from cellular to humoral immunity after oral pretreatment with antigen has also been observed in humans [14].

Indeed, recent studies indicate that oral administration of insulin affects the development of diabetes in NOD mice through the generation of cells that suppress the progression of insulitis [15, 16]. This probably occurs through the release of cytokines within the target organ which shift the balance from a Th1 to a Th2 pattern of cytokine expression [17]. However, the effects of such treatment were limited in that oral insulin only partially reduced the diabetes incidence in NOD mice. Moreover, the therapeutic dose range of oral insulin was very narrow [15]. It therefore seems desirable to potentiate the protective effects of oral insulin in NOD mice.

A previous study in an animal model of experimental autoimmune encephalomyelitis reported that the use of lipopolysaccharide (LPS) as adjuvant improved the outcome of oral antigen treatment [18]. Since oral endotoxin treatment may not be devoid of adverse effects, including the induction of autoantibody formation in the peritoneal cavity and lamina propria [19] we selected an LPS-free bacterial preparation as adjuvant. OM-89 is a protein extract of E scherichia coli containing mainly acidic glycoproteins of $10-300 \mathrm{kDa}$ molecular weight and less than 12 ppm LPS [20-22]. This compound is presently being used for adjuvant treatment of some infectious diseases and rheumatoid arthritis [20,23]. We also studied the mechanism of adjuvant action by determining cytokine production in vivo and in vitro.

\section{Materials and methods}

A nimals. Female NOD mice (nonobese diabetic, NOD/Bom) were purchased at Bomholtgard Breeding Centre (Ry, Denmark) and were kept under conventional conditions with standard diet for mice (Ssniff, Soest, Germany) with tap water ad libitum. "Principles of animal care" as well as the German Law on the Protection of Animals were followed.

E xperimental design. The NOD mice were allocated at random to four different treatment groups. Each group consisted of 36 animals. Mice were treated orally on 3 consecutive days per week for 5 weeks; starting at 7 weeks of age. Thereafter the treatment was reduced to 2 days of treatment every second week and stopped on day 140. The CTL group comprised sham treated animals receiving $0.9 \% \mathrm{NaCl}$ orally. The INS group was fed $0.9 \% \mathrm{NaCl}$, and the last treatment each week was $1 \mathrm{mg}$ of porcine insulin (kind gift of Hoechst, Frankfurt, Germany). The OM group received $200 \mathrm{mg} / \mathrm{kg}$ body weight of OM-89 (kind gift of Laboratoire OM, Geneva, Switzerland) and the last treatment each week was saline. Mice of the $\mathrm{OM}+\mathrm{INS}$ group received OM-89 and the last treatment each week was insulin.

Animals were monitored for glucosuria twice a week (Diabur; Boehringer Mannheim, Mannheim, Germany). Diabetes was confirmed after 3 consecutive days of glucosuria by hyperglycaemia $(\geq 14 \mathrm{mmol} / \mathrm{l})$, as determined in blood samples from non-fasted animals by the hexokinase method.

$\mathrm{H}$ istological analyses. On day 162 five non-diabetic animals per group were killed pancreas and gut were snap frozen in liquid nitrogen prechilled isopentane and stored at $-80^{\circ} \mathrm{C}$. Histological analysis and immunohistochemistry were done on acetonefixed cryostat sections. Sections for histology were stained with haematoxylin and eosin and were evaluated for mononuclear infiltration using the following semiquantitative rating system: score 0 , no periductular infiltrates and less than 5 immune cells peri- and intrainsular; score 1, periductular infiltrates only; score 2: periductular and peri-insular infiltrates only; score 3, intraislet infiltration of less than $20 \%$ of the islet area; score 4 , intraislet infiltration of more than $20 \%$ of the islet area. Buffers for cytokine-staining [3] were supplemented with $0.05 \%$ saponine (Sigma, Deisenhofen, Germany). Monoclonal antibodies against murine IL-4 and Il-2 were obtained from Pharmingen (clone BVD6-24G2 and clone S4B6; San Diego, Calif, USA). The total number of mononuclear cells surrounding and infiltrating islets was counted and the percentage of cytokine positive mononuclear cells per islet was determined. In each series of sections control stainings without first or second antibody were performed in order to check for background staining. In addition irrelevant rat monoclonal antibodies were used as negative controls.

mRNA analysis. Total RNA was isolated from whole small bowel by Trizol Reagent (Gibco BRL, Berlin, Germany). Determination and quantification of specific mRNA was performed by reverse transcriptase polymerase chain reaction (RT-PCR) as described elsewhere [3, 24]. Control experiments to check for linear relationship between amount of PCR product and amplification cycle number were performed. Specific primers were used for $\beta$-actin, interferon gamma (IFN $\gamma$ ), IL10, TGF $\beta$ (Clontech Laboratories Inc., Palo Alto, Calif., USA) and inducible nitric oxide synthase (iNOS) [25]. PCR products were labelled by hybridization to ${ }^{32} \mathrm{P}$ labelled probes binding at sides between the primer sequences. Signals were quantified by measuring the ${ }^{32} \mathrm{P}$ stimulated luminescence (PSL) by a phosphoimager. Relative PSL was calculated by normalization of the measured PSL to the strength of the $\beta$-actin signal [3].

M acrophage culture. The murine macrophage cell line IC21 was provided by ATCC (Rockville, Md., USA). Cells were cultured in petri dishes at $1 \times 10^{6} / \mathrm{ml}$ at $37^{\circ} \mathrm{C}$ in RPMI 1640 

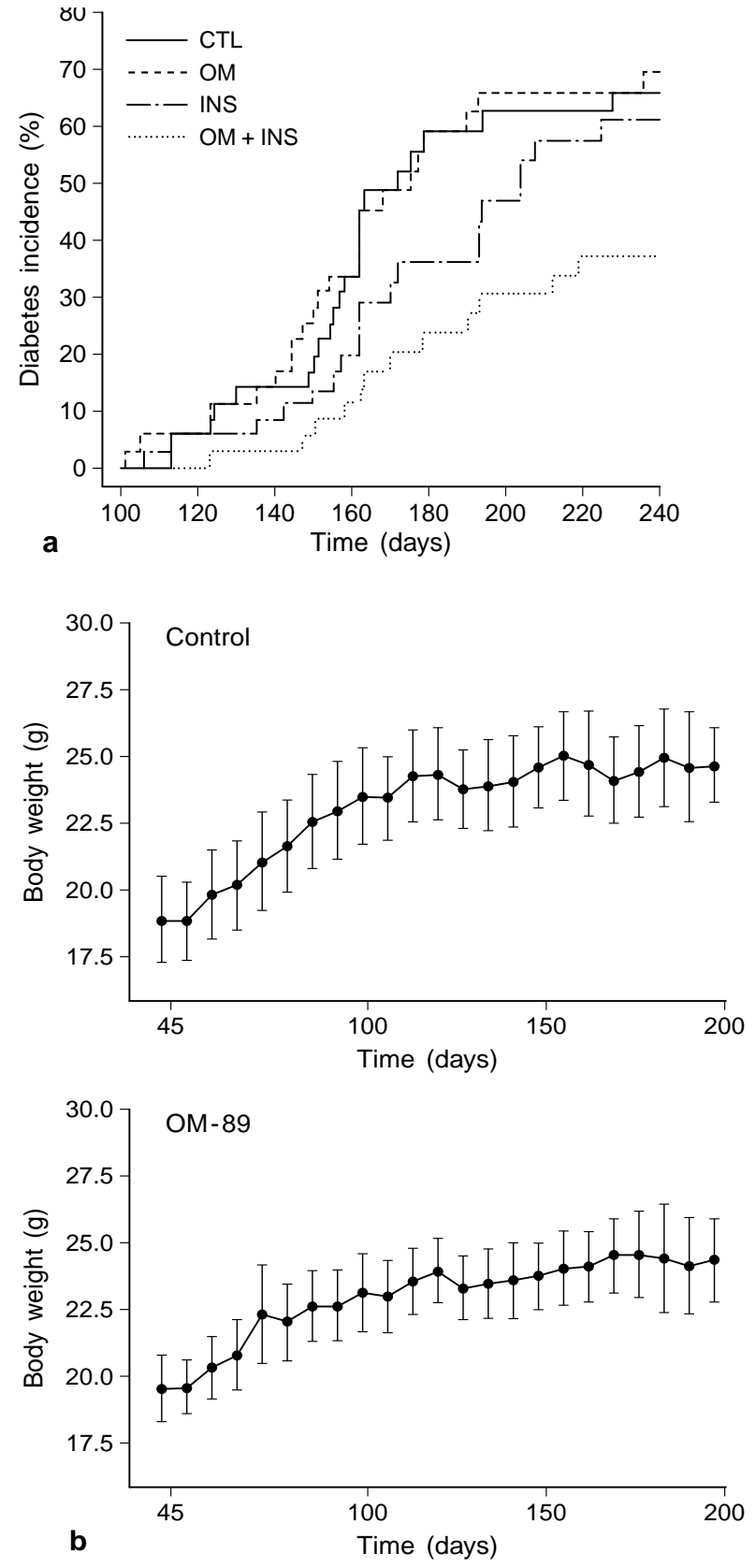

medium (Gibco, Heidelberg, Germany) supplemented with $10 \%(\mathrm{v} / \mathrm{v})$ fetal calf serum (FCS; Gibco) and $2 \mathrm{mmol} / \mathrm{l} \mathrm{L}$-glutamine. Cells were precultured for $24 \mathrm{~h}$ with OM-89 or sham treated with culture medium. After $24 \mathrm{~h}$ cells were washed with prewarmed RPMI 1640 and stimulated with LPS (E . coli 026:B6; Sigma, Deisenhofen, Germany), OM-89 or were sham treated. After $48 \mathrm{~h}$ supernatants were collected and nitrite and tumour necrosis factor alpha $(\mathrm{TNF} \alpha)$ levels were determined by Griess method [26] or TNF $\alpha$-bioassay [27], respectively, as described previously.

\section{Statistical analysis}

Diabetes incidence between groups was compared by the method of Kaplan and Meier. Immunohistochemistry data and RT-PCR analysis were compared by the Mann-Whitney-U
Fig. 1. Synergistic effect of oral insulin and OM-89 on diabetes prevention. NOD mice were treated orally with saline (CTL), insulin (INS), OM-89 (OM), and OM-89 and insulin (OM + INS) as described. Diabetes incidence was significantly reduced in the OM-89 and insulin treated group $(p=0.037)(\mathbf{a})$. Body weight did not differ significantly in all groups (b)
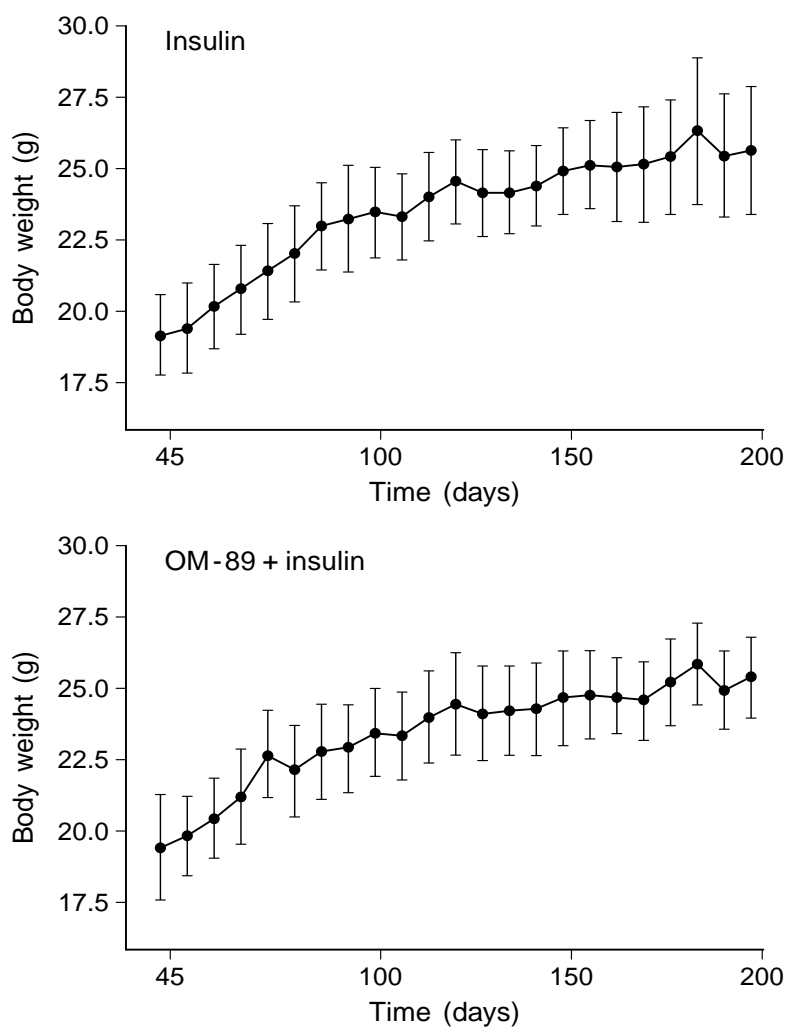

test. Cell culture experiment data were derived from three different experiments. Mean values were calculated and are presented with their standard deviation. Mean values were compared by two tailed Student's t-test. The level of significance was set at $p$ less than 0.05 .

\section{Results}

Synergistic effect of oral insulin and O M -89 on diabetes prevention. NOD mice were treated orally with $0.9 \% \mathrm{NaCl}$, insulin $(1 \mathrm{mg}), \mathrm{OM}-89$ (200 mg/kg body weight) or OM-89 and insulin as described. Diabetes incidence (Fig. 1 a) was significantly reduced in the group receiving OM-89 and insulin compared to the control group $(p<0.05)$. The insulin treated group 


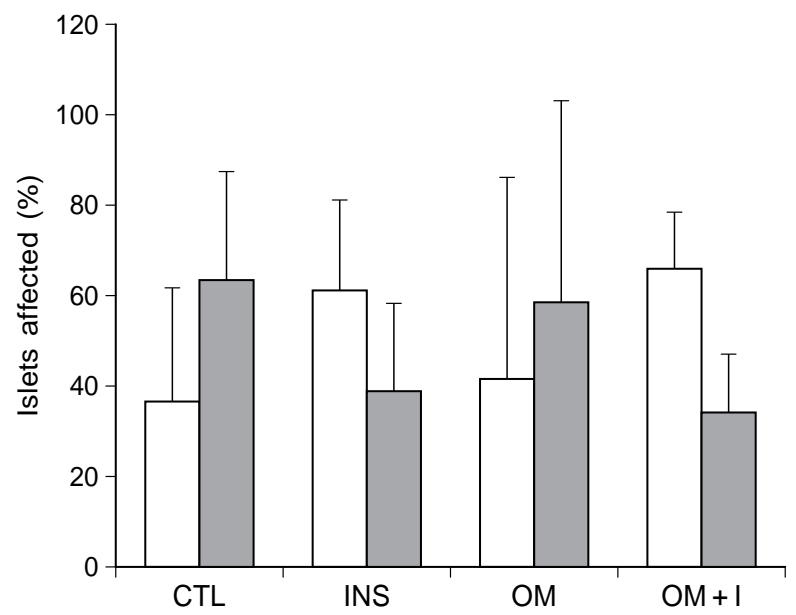

Fig. 2. Histological analysis. Pancreata of 5 NOD mice of each treatment group were excised on day 162 and histological analysis of the insulitis grade was performed. Per pancreas 15-25 islets were analysed for insulitis score. Percentage of islets with periinsular infiltration (scores $1-2) \square$, percentage of islets with intrainsular infiltration (scores $3-4)$. Means \pm SD, are given

showed a slightly delayed onset of diabetes, but reached the control level of diabetes incidence by the end of the experiment. Body weight did not differ significantly in all groups (Fig. 1 b).

$\mathrm{H}$ istological analysis. Semiquantitative analysis of insulitis was performed on day 162 . This date was chosen in order to determine insulitis quality at peak disease activity. In the control group $66 \%$ of islets showed advanced stages of islet inflammation (scores

Fig. 3. Impact on cytokine expression in islets. From the same pancreata described in Figure 2 consecutive cryostat sections were stained by immunohistochemistry for Th2 type cytokine IL-4 and Th1 type cytokine IL-2. The percentage of cytokine positive among total infiltrating cells (IL-4, IL-2) per islet was determined. Only islets with insulitis scores 3-4 (Fig. 2) were analysed, shown are mean scores of analysed islets. Statistical analysis was performed between indicated bars and control group

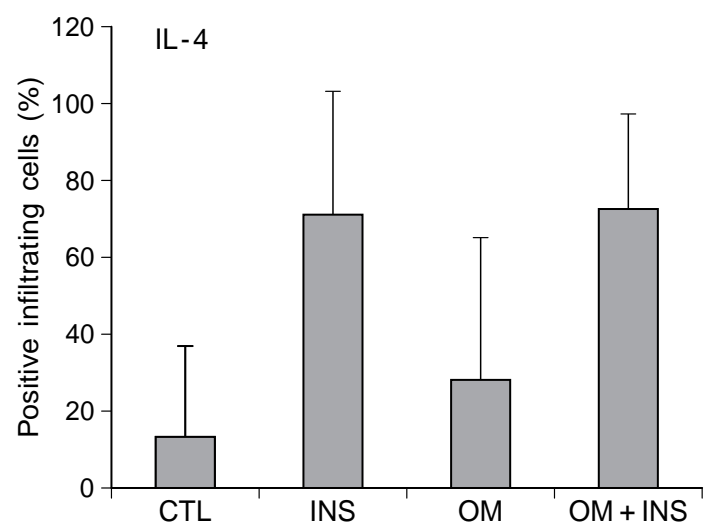

3-4). Treatment with OM-89 did not alter the histological picture, while the INS and OM + INS group showed a major decrease of severe insulitis (Fig.2).

Impact on cytokine expression in islets. Pancreas sections were analysed by immunohistochemistry for the Th2 type cytokine IL-4 and the Th1 type cytokine IL-2 in the islet infiltrate. In saline treated NOD mice less than $10 \%$ of infiltrating mononuclear cells were positive for IL-4 while $26 \%$ of cells expressed IL-2 (Fig. 3). Conversely, in the group receiving oral insulin the majority of cells expressed IL-4 $(p=0.015)$, while IL-2 positive cells were almost absent $(p=0.004)$. Treatment with OM-89 alone doubled the percentage of IL-4 positive cells (not significant) and did not affect the influx of IL-2 positive cells. The combination of OM-89 plus oral insulin led to a dominance of IL-4 positive cells in the infiltrate, as seen for oral insulin alone. However, there was no downregulation of IL-2 expression in the infiltrate (Fig. 3).

Impact on cytokine expression in the gut. Total gut RNA was analysed by RT-PCR for IFN $\gamma$, IL-10, TGF $\beta$ and iNOS (Fig. 4). Saline treated NOD mice showed little detectable IFN $\gamma$ mRNA in the gut, while substantial amounts of IL-10, TGF $\beta$ and iNOS were found. Interestingly, oral treatment with insulin alone did not affect cytokine and iNOS gene expression, except for an increase $(p<0.01)$ of IFN $\gamma$ mRNA. There was no such increase after OM-89 alone or after the combination of OM-89 and insulin. Conversely, treatment with OM-89 or with OM-89 plus insulin enhanced the Th2 related milieu of the gut by increasing IL-10 and TGF $\beta$ gene expression while suppressing iNOS mRNA levels.

D eviation of macrophage function by $0 \mathrm{M}-89$. Since the primary target of OM-89 among immune cells is the macrophage we determined when exposure of macrophages towards OM-89 altered macrophage functions. As shown in Figure 5, treatment of macrophages with OM-89 induced the release of $\mathrm{TNF} \alpha$ and nitrite. However, after contact with OM-89

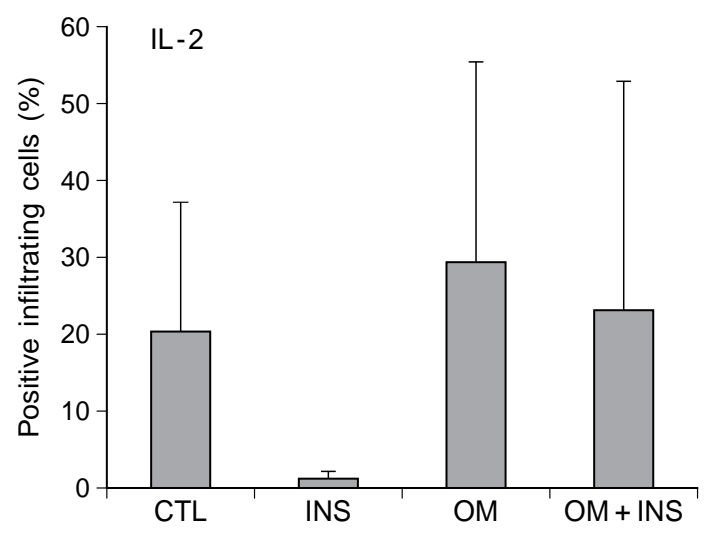




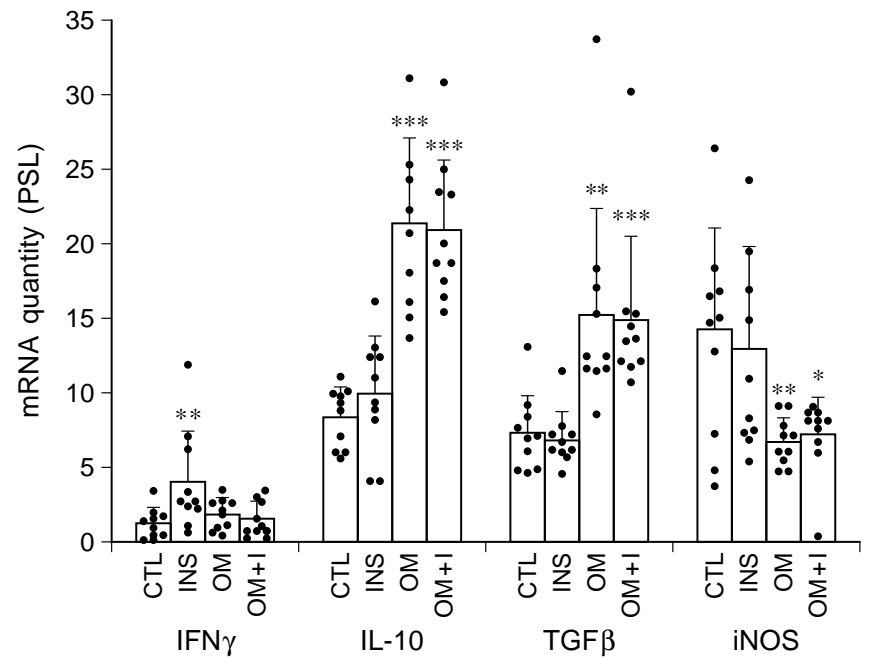

Fig. 4. Impact on cytokine expression in the gut. Five mice of each treatment group were killed on day 162 and proximal gut tissue was excised for RT-PCR analysis of cytokine mRNA. mRNA levels for IFN $\gamma$, IL-10, TGF $\beta$ and iNOS were determined by quantification of radiolabelling by phosphorstimulated luminiscence. The mRNA levels were calibrated to the amount of $\beta$-actin mRNA, which was set equal to one. Shown are two independent RT-PCR analysis per animal. Each point represents one RT-PCR of one animal. The columns represent means per group, bars indicate SD $(* p<0.05$, ** $p<0.01$, $* * * p<0.001$; compared to corresponding CTL-group)

macrophages became refractory towards restimulation with the potent macrophage activator LPS. This was observed for both inflammatory macrophage products, TNF $\alpha$ and nitrite (Fig. 5).

Fig. 5. Deviation of macrophage function by OM-89. IC-21 cells were cultured as described. $10^{6} / \mathrm{ml}$ cells were incubated with $1 \mathrm{mg} / \mathrm{ml} \mathrm{OM-89}(\mathrm{OM})$ or vehicle (-). After $24 \mathrm{~h}$ supernatants were removed, cells were washed with warm culture medium and stimulated with $1 \mathrm{mg} / \mathrm{ml} \mathrm{OM}-89$ (OM), $1 \mu \mathrm{g} / \mathrm{ml} \mathrm{LPS}$ (LPS) or sham treated (-). After an additional $24 \mathrm{~h}$ supernatants were collected for $\mathrm{TNF} \alpha$ and nitrite determination. Shown are means of three different experiments, bars indicate SD. Statistics were performed in comparison to LPS-stimulation after pretreatment with vehicle

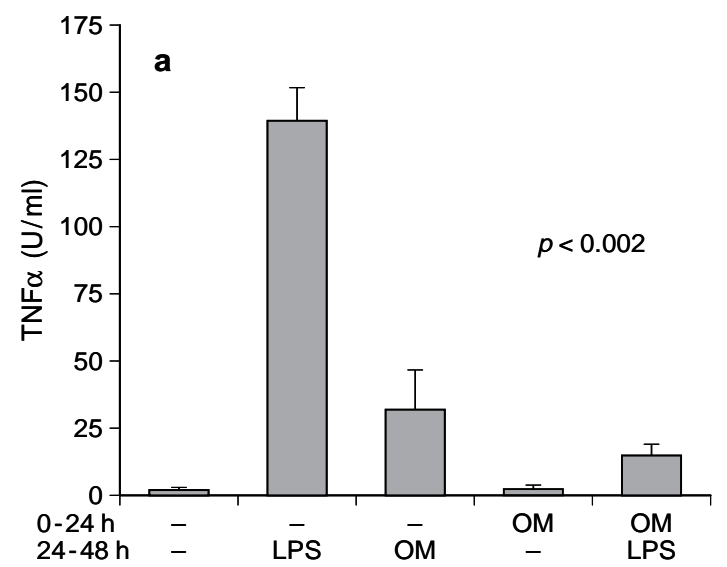

\section{Discussion}

Recent reports have shown a decrease in diabetes incidence in mice by approximately $50 \%$ after feeding a dose of $1 \mathrm{mg}$ porcine insulin [15]. In our study we used the same dose of insulin, but our protocol foresaw only one application of insulin per week, so that the total dosage of insulin applied over a period of 15 weeks was $50 \%$ smaller than previously [15]. This suboptimal protocol of oral insulin therapy was chosen in order to recognize better any potentiating effects of the bacterial preparation OM-89. Indeed, treatment of prediabetic NOD mice with oral insulin alone did not significantly suppress diabetes incidence although a transient small delay was observed. In contrast, mice that received the bacterial adjuvant prior to insulin showed a major delay in diabetes development and significant suppression of overt diabetes at any point of time beyond 157 days of age. This preventive effect was due to a synergistic effect of insulin and the adjuvant since oral administration of OM-89 alone did not alter the course of the disease.

Our results suggest that combination of oral antigen with a bacterial adjuvant can reduce the dosage of antigen without losing the therapeutic effect. This indicates that bacterial adjuvants can shift the therapeutic antigen dosage towards smaller amounts of antigen. Semiquantitative analysis of insulitis confirmed that the administration of OM-89 alone had not affected disease progression while there was a significant decrease of islets with intrainsulitis in mice receiving OM-89 plus insulin. Surprisingly, intrainsular infiltration was similarly reduced in the group receiving insulin alone. This may reflect the delay of diabetes development seen at the time of insulitis analyses (day 162).

A major difference between insulin and adjuvant plus insulin groups became apparent when staining cytokine expression within affected islets. We chose the two cytokines IL-2 and IL-4 as indicators of Th1 or Th2 reactivity, since these gave the strongest signal for either type of immunoreactivity in islets of

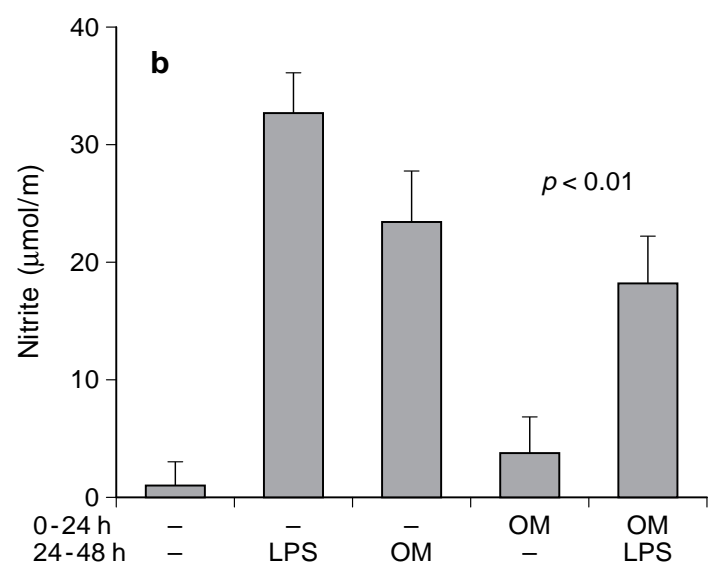


NOD mice with spontaneous development of diabetes while in cyclophosphamide accelerated diabetes development IFN $\gamma$ is the predominant Th1 cytokine [3]. Insulitis in sham treated control mice was characterized by a clear dominance of IL-2 positive over IL-4 positive cells, indicating a bias towards Th1 type reactivity. Treatment with oral insulin alone completely reversed the immunoregulatory balance, leading to a dramatic increase of IL-4 positive infiltrate while IL-2 positive cells were barely detectable. These changes correspond to those described by Hancock et al. [17] for the more intense oral insulin dosing protocol.

Upregulation of IL-4 positive cells in the infiltrate was also seen in the group receiving adjuvant plus insulin. However, there was no decrease in the percentage of IL-2 positive cells. Since the combined treatment with the bacterial preparation and insulin suppressed the progression towards diabetes, it is conceivable that the release of IL-2 in conjunction with IL-4 is superior in limiting destructive insulitis than IL-4 alone. One major protective effect of IL-2 in the presence of IL-4 may be its growth promoting, anti-apoptotic effect. Indeed, Zhang et al. [28] recently showed proliferation of $\mathrm{Th} 2$ effector cells and prevention of apoptotic cell death in the presence of IL-2 while prolonged, dramatic expansion of Th1 cells occurred during IL-2 deficiency. Impaired IL-2 production has been reported to be a characteristic of IDDM and its animal models [29-32]. A protective effect of IL-2 injections was reported in a BB rat line with high diabetes risk [33] and transgenic mice expressing IL-2 under the rat insulin promotor developed apparently benign insulitis because progression to diabetes was rarely observed [34]. We conclude that IL-2 may not only function as a Th1 cytokine but may also sustain ongoing Th2 responses in the presence of IL-4.

The different cytokine pattern induced by insulin or by insulin plus adjuvant indicates some variability in the outcome of oral tolerization. Hence, it is not surprising that oral antigen dosing was recently even found in settings particularly to exacerbate autoimmune disease $[35,36]$.

In order to understand how the adjuvant might have affected the gut mucosa immune response to oral insulin we analysed the cytokine expression pattern in the gut. Because IL-10 and TGF $\beta$ are the major anti-inflammatory Th2 type cytokines, but not IL-4 [3], the former two were analysed, in parallel with IFN $\gamma$ as a key Th1 cytokine in the gut. As marker of macrophage and/or epithelial cell activation iNOS mRNA was determined. The normal NOD gut showed little IFN $\gamma$ and a predominance of IL-10 and TGF $\beta$ mRNA. Interestingly, substantial amounts of iNOS mRNA were found. IL-10 gene expression was increased by treatment with OM-89 while insulin alone failed to induce IL-10. Similarly, TGF $\beta$, a cytokine typical for tolerance induction [24, 37, 38], was elevated by OM-89 but not by insulin administration. Conversely, iNOS mRNA was decreased by OM-89 treatment while oral insulin alone did not downregulate iNOS gene expression. Interestingly, IFN $\gamma$ was slightly elevated in the gut of the insulin treated group.

In summary, the cytokine profile of the gut shows a clear deviation of the immune balance to Th2 type reactivity by oral treatment with bacterial adjuvant OM-89 but not by oral application of insulin alone. It is known that the cytokine milieu present during antigen presentation is crucial in driving $\mathrm{T}$ cell responses compared to Th1 or Th2. In the presence of IL-10 and TGF $\beta$, responses to oral insulin will be biased towards Th2. Potentiation of the apparent Th2 bias of the gut by OM-89 can be expected to drive Tcell responses even more strongly towards Th2. This view is in accord with the observed synergism of OM-89 and suboptimal insulin dosage. It remains to be determined whether the disease suppressing effects of optimal oral antigen doses can be further enhanced by adjuvant.

What is the target of adjuvant action? It has been reported that the immunostimulatory peptides of OM-89 interact with macrophages rather than with $T$ cells [39]. Hence, mucosal antigen presenting cells are probably the major targets of OM-89 in the gut. A change in the functional state of antigen presenting cells in the gut might affect the outcome of antigen presentation. Bacterial immunostimulatory compounds such as OM-89 are known to induce the release of proinflammatory Th1 type mediators in macrophages [40] or other antigen presenting cells [41]. However, as we show here, exposure to the bacterial adjuvant prevented restimulation and further release of proinflammatory mediators. This desensitizing action of OM-89 seen in vitro is reminiscent of the phenomenon of LPS tolerance [42] and fits well with the suppression of iNOS mRNA observed here in the gut of OM-89 treated NOD mice.

We conclude that the protective effect of oral insulin on the progression of insulitis and the development of overt diabetes in NOD mice can be potentiated by pretreatment with the bacterial adjuvant OM-89. This effect correlates with enhanced Th2 cytokine and decreased iNOS gene expression, probably as a consequence of local downregulation of proinflammatory Th1 type mediators by repeated exposure to the adjuvant.

A cknowledgements. We thank Dr. Helga Rothe for help with RT-PCR and M. Rinker for technical assistance. This work was supported by the Bundesministerium für Bildung, Wissenschaft, Forschung und Technologie, the Bundesminister für Gesundheit and the Minister für Wissenschaft und Forschung des Landes Nordrhein-Westfalen. We are grateful to Dr. A. Kollar for a donation in support of the oral tolerance program. 


\section{References}

1. Castano L, Eisenbarth GS (1990) Type-I diabetes: a chronic autoimmune disease of human, mouse, and rat. Annu Rev Immunol 8: 647-679

2. Rossini A, Mordes JP, Greiner DL (1989) The pathogenesis of autoimmune diabetes mellitus. Curr Opinion Immunol 2: 598-603

3. Rothe H, Faust A, Schade U, Kleemann R, Bosse G, Hibino T, Martin S, Kolb H (1994) Cyclophosphamide treatment of female non-obese diabetic mice causes enhanced expression of inducible nitric oxide synthase and interferon-gamma, but not of interleukin-4. Diabetologia 37: 1154-1158

4. Rabinovitch A (1994) Immunoregulatory and cytokine imbalances in the pathogenesis of IDDM-therapeutic intervention by immunostimulation? Diabetes 43: 613-621

5. Pennline KJ, Roque-Gaffney E, Monahan M (1994) Recombinant human IL-10 prevents the onset of diabetes in NOD mice. Clin Immunol and Immunopathol 71: 169-175

6. Rapoport MJ, Jaramillo A, Zipris D et al. (1993) IL-4 reverses T-cell proliferative unresponsiveness and prevents the onset of diabetes in NOD mice. J Exp Med 178: 87-99

7. Shehadeh NN, LaRosa F, Lafferty KJ (1993) Altered cytokine activity in adjuvant inhibition of autoimmune diabetes. J Autoimm 6: 291-300

8. Muir A, Schatz D, Maclaren N (1993) Antigen specific immunotherapy: oral tolerance and subcutaneous immunization in the treatment of insulin-dependent diabetes. Diabetes Metab Rev 9: 279-287

9. Thompson HSG, Staines NA (1986) Gastric administration of type II collagen delays the onset and severity of collagen-induced arthritis in rats. Clin Exp Immunol 64: 581-586

10. Nagler-Anderson C, Bober LA, Robinson ME, Siskind GW, Thorbecke FJ (1986) Suppression of type II collageninduced arthritis by intragastric administration of soluble type II collagen. Proc Natl Acad Sci USA 83: 7443-7446

11. Higgins P, Weiner HL (1988) Suppression of experimental autoimmune encephalomyelitis by oral administration of myelin basic protein and its fragments. J Immunol 140: 440-445

12. Miller A, Lider O, Weiner HL (1991) Antigen driven bystander suppression following oral administration of antigens. J Exp Med 174: 791-798

13. Weiner HL, Friedmann A, Miller A et al. (1994) Oral tolerance: immunologic mechanisms and treatment of animal and human organ-specific autoimmune diseases by oral administration of autoantigens. Annu Rev Immunol 12: 809837

14. Husby S, Mestecky J, Moldoveanu Z, Holland S, Elson CH (1994) Oral tolerance in humans. T cell but not B cell tolerance after antigen feeding. J Immunol 152: 4663

15. Zhang ZJ, Davidson L, Eisenbarth G, Weiner HL (1991) Suppression of diabetes in nonobese diabetic mice by oral administration of porcine insulin. Proc Nat Acad Sci USA 88: $10252-10256$

16. Bergerot I, Fabien N, Maguer V, Thivolet C (1994) Oral administration of human insulin to NOD mice generates CD4 (+) T cells that suppress adoptive transfer of diabetes. J Autoimmun 7: 655-663

17. Hancock WW, Polanski M, Zhang J, Blogg N, Weiner HL (1995) Suppression of insulitis in non-obese Diabetic (NOD) mice by oral insulin administration is associated with selective expression of interleukin- 4 and -10 , transforming growth factor-b, and prostaglandin-E. Am J Pathol 147: 1193-1199
18. Khoury SJ, Hancock WW, Weiner HL (1992) Oral tolerance to myelin basic protein and natural recovery from experimental autoimmune encephalomyelitis are associated with downregulation of inflammatory cytokines and differential upregulation of transforming growth factor beta, interleukin 4, and prostaglandin E expression in the brain. $\mathrm{J}$ Exp Med 176: 1355-1364

19. Murakami M, Tsubata T, Shinkura R et al. (1994) Oral administration of lipopolysaccharides activates B-1 cells in the peritoneal cavity and lamina propria of the gut and induces autoimmune symptoms in an autoantibody transgenic mouse. J Exp Med 180: 111-121

20. Peters DH, Goa KL (1994) OM-8980 - an initial review of its pharmacology and therapeutic potential in rheumatoid arthritis. Clin Immunother 2: 65-67

21. Polla BS, Baladi S, Fuller K, Rook G (1995) Presence of hsp65 in bacterial extracts (OM-89): a possible mediator of orally-induced tolerance? Experientia 51: 775-779

22. Kiesel U (1994) Prevention of diabetes in BB rats by an E. coli extract (OM-89). Int J Immunopathol Pharmacol 7: 193-199

23. Tammen $\mathrm{H}$, The German urinary tract infection study group (1990) Immunobiotherapy with Uro-Vaxom in recurrent urinary tract infection. Brit J Urol 65: 6-9

24. Rothe H, Burkart V, Faust A, Kolb H (1996) IL-12 gene expression is associated with rapid development of diabetes mellitus in non-obese diabetic mice. Diabetologia 39: 119122

25. Kleemann R, Rothe H, Kolb-Bachofen V, Xie Q-W, Nathan C, Martin S, Kolb H (1993) Transcription and translation of inducible nitric oxide synthase in the pancreas of prediabetic BB rats. FEBS Lett 328: 9-12

26. Kröncke K-D, Kolb-Bachofen V, Berschick B, Burkart V, Kolb H (1991) Activated macrophages kill pancreatic syngeneic islet cells via arginine-dependent nitric oxide generation. Biochem Biophys Res Commun 175: 752-758

27. Unglaub R, Maxeiner B, Thoma B, Pfizenmeier K, Scheurich $\mathrm{P}$ (1987) Downregulation of tumor necrosis factor sensitivity via modulation of TNF binding capacity by protein kinase C activators. J Exp Med 166: 1788-1797

28. Zhang X, Giangreco L, Broome HE, Dargan CM, Swain SL (1995) Control of CD4 effector fate: transforming growth factor b1 and interleukin 2 synergize to prevent apoptosis and promote effector expansion. J Exp Med 182: 699-709

29. Kay WA, Adri MNS, Soeldner JS et al. (1986) Acquired defect in interleukin-2 production in patients with type I diabetes mellitus. New Engl J Med 315: 920-925

30. Lang F, Pogu S, Maurel C, Charbonnel B, Sai P (1987) Production of and response to interleukin 2 by blood mononuclear cells from some type I diabetic patients. Diabetes Metabolism 13: 37-43

31. Prud'homme GJ, Fuks A, Colle E, Seemayer TA, Guttmann RD (1984) Immune dysfunction in diabetes-prone $\mathrm{BB}$ rats. Interleukin 2 production and other mitogen-induced responses are suppressed by activated macrophages. J Exp Med 159:463-478

32. Zier KS, Leo MM, Spielmann RS, Baker L (1984) Decreased synthesis of interleukin 2 (IL-2) in insulin-dependent diabetes mellitus. Diabetes 33:552-555

33. Zielaseck J, Burkart V, Naylor P, Goldstein A, Kiesel U, Kolb H (1990) Interleukin-2-dependent control of disease development in spontaneously diabetic BB rats. Immunology 69:209-214

34. von Herrath MG, Allison J, Miller JFAP, Oldstone MBA (1995) Focal expression of interleukin-2 does not break unresponsiveness to "self" (viral) antigen expressed in b cells 
but enhances development of autoimmune disease (diabetes) after initiation of an anti-self immune response. J Clin Invest 95: 477-485

35. Blanas E, Carbone FR, Allison J, Miller JFAP, Heath WR (1996) Induction of autoimmune diabetes by oral administration of autoantigen. Science 274: 1707-1709

36. Meyer AL, Benson JM, Gienapp IE, Cox KL, Whitacre CC (1996) Suppression of murine chronic relapsing experimental autoimmune encephalomyelitis by the oral administration of myelin basic protein. J Immunol 157: 4230-4238

37. Severn A, Xu D, Doyle J et al. (1993) Pre-exposure of murine macrophages to lipopolysaccharide inhibits the induction of nitric oxide synthase and reduces leishmanicidal activity. Eur J Immunol 23: 1711-1714

38. Fukaura H, Kent SC, Pietrusewicz MJ, Khoury SJ, Weiner HL, Hafler DA (1996) Induction of circulating myelin basic protein and proteolipid protein-specific transforming growth factor-1-secreting Th3 T cells by oral administration of myelin in multiple sclerosis patients. J Clin Invest 98: 70 77

39. Bessler WG, Beck P, Konetznick U et al. (1991) Biological activity of bacterial surface components. Immunogenicity and immunomodulatory properties of bacterial extract from Escherichia coli. Arzneim-Forsch/Drug Res 41: 274279

40. Bottex C, Cristau B, Corazza JL, Mougin B, Fontanges R (1988) Effects of two bacterial extracts, OM-89 and Broncho-Vaxom, on IL-1 release and metabolic activity of a murine macrophage cell-line. Int J Immunotherapy 4:203-212

41. Pham TV, Kreis B, Corradin-Betz S, Bauer J, Mauel J (1990) Metabolic and functional stimulation of lymphocytes and macrophages by an Escherichia coli extract (OM-89): in vitro studies. J Biol Resp Mod 9: 231-240

42. Ziegler-Heitbrock HWL (1995) Molecular mechanism in tolerance to lipopolysaccharide. J Inflamm 45: 13-26 